\title{
Recursive approach of optimal Kalman filtering problem for multiparameter singularly perturbed systems
}

\author{
H. MUKAIDANI* \\ Graduate School of Education, Hiroshima University, \\ 1-1-1 Kagamiyama, Higashi-Hiroshima 739-8524, Japan \\ (Received 11 November 2002; revised 24 June 2004; accepted 15 October 2004)
}

\begin{abstract}
In this paper, the optimal Kalman filtering problem for multiparameter singularly perturbed systems is considered. In order to obtain the filter gain, the solution of the multiparameter algebraic Riccati equations (MAREs) is needed. The main contributions in this paper are to propose a new recursive algorithm for solving the MARE and to establish sufficient conditions related to the convergence property of the proposed algorithm. Using the recursive algorithm, it is shown that the solution of the MARE converges to a positive semidefinite stabilizing solution with the rate of convergence of $O\left(\|\mu\|^{i}\right)$. Moreover, it is proved that the mean square error via the proposed high-order filter attain, the $O\left(\|\mu\|^{2 i+1}\right)$ approximation compared with the optimal filter.
\end{abstract}

\section{Introduction}

Filtering problems for the multiparameter singularly perturbed systems (MSPSs) have been investigated extensively (see for example, Coumarbatch and Gajić (2000), and reference therein). The multimodelling problems arise in large-scale dynamic systems. For example, the multimodel situation in practice is illustrated by the passenger car model (Coumarbatch and Gajić 2000). In order to obtain the optimal solution to the multimodelling problems, the multiparameter algebraic Riccati equation (MARE) needs to be solved. Various reliable approaches to the theory of the algebraic Riccati equation (ARE) have been well documented in many publications (see for example Laub 1979). One of the approaches is the invariant subspace approach which is based on the Hamiltonian matrix (Laub 1979). However, there is no guarantee of symmetry for the computed solution if the ARE is ill conditioned (Laub 1979). Note that it is very difficult to solve the MARE owing to high dimension and numerical stiffness (Coumarbatch and Gajić 2000).

A popular approach to deal with the MSPS is the two-time-scale design method (see for example,

*Corresponding author. Email: mukaida@hiroshima-u.ac.jp
Khalil and Kokotović (1979), Kokotović et al. (1986), Mukaidani (2003) and Mukaidani et al. (2003)). However, it is known from the work of Coumarbatch and Gajić (2000) that an $O(\|\mu\|)$ (where $\mu:=\left[\begin{array}{ll}\varepsilon_{1} & \varepsilon_{2}\end{array}\right]$ ) accuracy is very often not sufficient. In particular, it is important to note that the required solution must be obtained perfectly because the corresponding bound on the steady-state mean square error tends to infinity when $\|\mu\| \rightarrow 0$. Furthermore, in order to construct the filter, knowledge of the parameters is needed. Therefore, special effort must be made to solve the MARE as long as information on these parameters is available.

Recently, the exact slow-fast decomposition method for solving the MARE of the MSPS has been proposed (Coumarbatch and Gajic 2000). However, these results are restricted to the MSPS such that the Hamiltonian matrices for the fast subsystems have no eigenvalues in common (see assumption 5 of the paper by Coumarbatch and Gajić (2000)). More recently, the Kleinman algorithm for solving the MARE has been established (Mukaidani et al. 2002a,b). However, in order to reduce the computational workspace, another algorithm such as fixed-point iterations is needed. As another important algorithm in the work of Mukaidani et al. (2002d), the recursive algorithm for the solution of the regulator-type MARE has been 
proposed. On the other hand, even though the $H_{\infty}$ filtering problem via the recursive algorithm has been studied (Mukaidani et al. 2002c), the recursive algorithm for solving the MARE corresponding to the Kalman filtering problem has not been investigated. In particular, the existence condition for the solution of the MARE and its asymptotic property have not been studied. Furthermore, so far, the loss of steady-state mean square error between the optimal filter and the proposed filters that are based on the useful numerical methods such as the recursive technique or the exact decomposition technique has not been established.

In this paper, the optimal Kalman filtering problem for the MSPS is studied. In particular, two-parameter singularly perturbed systems (TSPSs) are considered. The results obtained are valid for the steady state. First the uniqueness and boundedness of the solution to such a MARE are investigated and its asymptotic structure and the existence condition are derived. The proof of the existence of the solution to the MARE with asymptotic expansion is obtained by an implicit function theorem (Gajic et al. 1990). The main result of this paper is to propose a new recursive algorithm for solving the MARE and to find the sufficient conditions regarding the convergence of the recursive algorithm by using the reduced-order ARE. It is important to note that the sufficient conditions derived here are independent of the small perturbation parameter $\mu$. It is also proved that the solution of the MARE converges to a positive semidefinite stabilizing solution with the rate of convergence of $O\left(\|\mu\|^{i+1}\right)$, where $i$ is the iteration number. As another important feature, the assumption that the Hamiltonian matrices for the fast subsystems have no eigenvalues in common is not needed. Thus, the new results are applicable to a more realistic MSPS. Moreover, it is shown that the highorder approximate Kalman filter achieves a performance which is $O\left(\|\mu\|^{2 i+1}\right)$ close to the optimal mean square error. It is worth pointing out that the feature of the $O\left(\|\mu\|^{2 i+1}\right)$ suboptimality is established for the first time for the optimal filtering problem of the MSPS.

\subsection{Notation}

The superscript $\mathrm{T}$ denotes matrix transpose. $\operatorname{det} L$ denotes the determinant of the square matrix $L$. $I_{n}$ denotes the $n \times n$ identity matrix. $\|\cdot\|$ denotes its Euclidean norm for a matrix. $\otimes$ denotes the Kronecker product (Magnus and Neudecker 1999). $U_{l m}$ denotes a permutation matrix in the Kronecker matrix sense (Magnus and Neudecker 1999) such that $U_{l m} \operatorname{vec} M=\operatorname{vec} M^{\mathrm{T}}, M \in \mathbf{R}^{l \times m} . E\{\cdot\}$ denotes the expectation operator. $\operatorname{Tr}$ denotes the trace operator.

\section{Optimal Kalman filtering problem}

Let us consider the following linear time-invariant MSPS (TSPS):

$$
\begin{aligned}
\dot{x}_{0} & =A_{00} x_{0}+A_{01} x_{1}+A_{02} x_{2}+D_{01} w_{1}+D_{02} w_{2}, \\
\varepsilon_{1} \dot{x}_{1} & =A_{10} x_{0}+A_{11} x_{1}+D_{11} w_{1}, \\
\varepsilon_{2} \dot{x}_{2} & =A_{20} x_{0}+A_{22} x_{2}+D_{22} w_{2},
\end{aligned}
$$

with the corresponding measurements

$$
y_{j}=C_{j 0} x_{0}+C_{j j} x_{j}+v_{j}, \quad j=1,2,
$$

where $x_{j} \in \mathbf{R}^{n_{j}}, j=0,1,2$, are the state vectors, $y_{j} \in \mathbf{R}^{p_{j}}$, $j=0,1,2$, are the system measurements, $w_{j} \in \mathbf{R}^{q_{j}}$, $j=1,2$, and $v_{j} \in \mathbf{R}^{r_{j}}, j=1,2$, are zero mean white Gaussian noise processes with the following joint covariance matrix, respectively:

$$
\begin{gathered}
W:=E\left\{\left[\begin{array}{l}
w_{1} \\
w_{2}
\end{array}\right]\left[\begin{array}{ll}
w_{1}^{\mathrm{T}} & w_{2}^{\mathrm{T}}
\end{array}\right]\right\}=\left[\begin{array}{cc}
W_{11} & 0 \\
0 & W_{22}
\end{array}\right], \\
V:=E\left\{\left[\begin{array}{l}
v_{1} \\
v_{2}
\end{array}\right]\left[\begin{array}{ll}
v_{1}^{\mathrm{T}} & v_{2}^{\mathrm{T}}
\end{array}\right]\right\}=\left[\begin{array}{cc}
V_{11} & 0 \\
0 & V_{22}
\end{array}\right], \\
E\left\{\left[\begin{array}{l}
w_{1} \\
w_{2}
\end{array}\right]\left[\begin{array}{ll}
v_{1}^{\mathrm{T}} & v_{2}^{\mathrm{T}}
\end{array}\right]\right\}=\left[\begin{array}{ll}
0 & 0 \\
0 & 0
\end{array}\right] .
\end{gathered}
$$

All the matrices are constant matrices of appropriate dimensions. Moreover, $\varepsilon_{1}$ and $\varepsilon_{2}$ are two small positive singular parameters of the same order of magnitude such that

$$
0<k_{1} \leqslant \alpha \equiv \frac{\varepsilon_{1}}{\varepsilon_{2}} \leqslant k_{2}<\infty
$$

That is, it is assumed that the ratio of $\varepsilon_{1}$ to $\varepsilon_{2}$ is bounded by some positive constants $k_{j}, j=1,2$.

In this paper the design method of the Kalman filter gain for estimating the system states $x_{j}$ is established under the following basic assumption.

Assumption 1: The limit of $\alpha$ exists as $\varepsilon_{1}$ and $\varepsilon_{2}$ tend to zero (Khalil and Kokotović 1979, Kokotović et al. 1986), that is

$$
\bar{\alpha}=\lim _{\substack{\varepsilon_{1} \rightarrow 0^{+} \\ \varepsilon_{2} \rightarrow 0^{+}}} \alpha .
$$

The above assumption guarantees the existence for the limiting solution of the MARE given later. 
The optimal Kalman filter of (1) and (2) is given by (Coumarbatch and Gajić 2000)

$$
\begin{aligned}
\dot{\xi}_{0} & =A_{00} \xi_{0}+A_{01} \xi_{1}+A_{02} \xi_{2}+F_{01} \eta_{1}+F_{02} \eta_{2}, \\
\varepsilon_{1} \dot{\xi}_{1} & =A_{10} \xi_{0}+A_{11} \xi_{1}+F_{11} \eta_{1}+F_{12} \eta_{2}, \\
\varepsilon_{2} \dot{\xi}_{2} & =A_{20} \xi_{0}+A_{22} \xi_{2}+F_{21} \eta_{1}+F_{22} \eta_{2}, \\
\eta_{j} & =y_{j}-C_{j 0} \xi_{0}-C_{j j} \xi_{j}, \quad j=1,2,
\end{aligned}
$$

where $\xi_{j} \in \mathbf{R}^{n_{j}}, j=0,1,2$, are the state estimates and $\eta_{j} \in \mathbf{R}^{p_{j}}, j=0,1,2$, are the prediction errors.

The filter gain matrices that are represented as $F_{0 j}$ and $F_{j j}, j=1,2$, are obtained from

$$
F_{e}:=\left[\begin{array}{cc}
F_{01} & F_{02} \\
\varepsilon_{1}^{-1} F_{11} & \varepsilon_{1}^{-1} F_{12} \\
\varepsilon_{2}^{-1} F_{21} & \varepsilon_{2}^{-1} F_{22}
\end{array}\right]=Y_{e} C^{\mathrm{T}} V^{-1} .
$$

$Y_{e}$ satisfies the MARE

$$
A_{e} Y_{e}+Y_{e} A_{e}^{\mathrm{T}}-Y_{e} S Y_{e}+U_{e}=0,
$$

with

$$
\begin{aligned}
A_{e} & :=\left[\begin{array}{ccc}
A_{00} & A_{01} & A_{02} \\
\varepsilon_{1}^{-1} A_{10} & \varepsilon_{1}^{-1} A_{11} & 0 \\
\varepsilon_{2}^{-1} A_{20} & 0 & \varepsilon_{2}^{-1} A_{22}
\end{array}\right], \\
D_{e} & :=\left[\begin{array}{cc}
D_{01} & D_{02} \\
\varepsilon_{1}^{-1} D_{11} & 0 \\
0 & \varepsilon_{2}^{-1} D_{22}
\end{array}\right], \quad C:=\left[\begin{array}{ccc}
C_{10} & C_{11} & 0 \\
C_{20} & 0 & C_{22}
\end{array}\right], \\
S & :=C^{\mathrm{T}} V^{-1} C=\left[\begin{array}{ccc}
S_{00} & S_{01} & S_{02} \\
S_{01}^{\mathrm{T}} & S_{11} & 0 \\
S_{02}^{\mathrm{T}} & 0 & S_{22}
\end{array}\right], \\
U_{e} & :=D_{e} W D_{e}^{\mathrm{T}}=\left[\begin{array}{ccc}
U_{00} & \varepsilon_{1}^{-1} U_{01} & \varepsilon_{2}^{-1} U_{02} \\
\varepsilon_{1}^{-1} U_{01}^{\mathrm{T}} & \varepsilon_{1}^{-2} U_{11} & 0 \\
\varepsilon_{2}^{-1} U_{02}^{\mathrm{T}} & 0 & \varepsilon_{2}^{-2} U_{22}
\end{array}\right] .
\end{aligned}
$$

Since the matrices $A_{e}$ and $D_{e}$ contain the term of $\varepsilon_{j}^{-1}$ order, the solution $Y_{e}$ of the MARE (8), if it exists, must contain terms of $\varepsilon_{j}$ order. Taking this fact into account, the purpose of this paper is to look for the solution $Y_{e}$ for the MARE (8) with the structure

$$
Y_{e}:=\left[\begin{array}{ccc}
Y_{00} & Y_{10}^{\mathrm{T}} & Y_{20}^{\mathrm{T}} \\
Y_{10} & \varepsilon_{1}^{-1} Y_{11} & \left(\varepsilon_{1} \varepsilon_{2}\right)^{-1 / 2} Y_{21}^{\mathrm{T}} \\
Y_{20} & \left(\varepsilon_{1} \varepsilon_{2}\right)^{-1 / 2} Y_{21} & \varepsilon_{2}^{-1} Y_{22}
\end{array}\right] \in \mathbf{R}^{N \times N},
$$

where $N=n_{0}+n_{1}+n_{2}, \quad Y_{00}=Y_{00}^{\mathrm{T}}, \quad Y_{11}=Y_{11}^{\mathrm{T}} \quad$ and $Y_{22}=Y_{22}^{\mathrm{T}}$.
In order to avoid the ill conditioning due to the large parameter $\varepsilon_{j}^{-1}$ which is included in the MARE (8), let us introduce the following useful result (Mukaidani et al. 2002d).

The MARE (8) is equivalent to the following generalized multiparameter algebraic Riccati equation (GMARE) (9a):

$$
\begin{aligned}
\mathcal{F}(Y) & :=A Y^{\mathrm{T}}+Y A^{\mathrm{T}}-Y S Y^{\mathrm{T}}+U=0, \\
Y_{e} & =Y^{\mathrm{T}} \Phi_{e}^{-1}=\Phi_{e}^{-1} Y,
\end{aligned}
$$

where

$$
\begin{aligned}
\Phi_{e}: & =\left[\begin{array}{ccc}
I_{n_{0}} & 0 & 0 \\
0 & \varepsilon_{1} I_{n_{1}} & 0 \\
0 & 0 & \varepsilon_{2} I_{n_{2}}
\end{array}\right], \\
A & :=\left[\begin{array}{ccc}
A_{00} & A_{01} & A_{02} \\
A_{10} & A_{11} & 0 \\
A_{20} & 0 & A_{22}
\end{array}\right],
\end{aligned}
$$

$$
\begin{aligned}
& U:= {\left[\begin{array}{ccc}
U_{00} & U_{01} & U_{02} \\
U_{01}^{\mathrm{T}} & U_{11} & 0 \\
U_{02}^{\mathrm{T}} & 0 & U_{22}
\end{array}\right], } \\
& Y:=\left[\begin{array}{ccc}
Y_{00} & Y_{10}^{\mathrm{T}} & Y_{20}^{\mathrm{T}} \\
\varepsilon_{1} Y_{10} & Y_{11} & \alpha^{1 / 2} Y_{21}^{\mathrm{T}} \\
\varepsilon_{2} Y_{20} & \alpha^{-1 / 2} Y_{21} & Y_{22}
\end{array}\right] .
\end{aligned}
$$

\section{The multiparameter algebraic Riccati equation}

In the following analysis, the basic assumptions are needed. Specifically, in order to guarantee the existence of the reduced-order ARE and its standard stabilizability and the detectability conditions when $\|\mu\|:=$ $\left(\varepsilon_{1}^{2}+\varepsilon_{2}^{2}\right)^{1 / 2} \rightarrow 0^{+}$, assumptions 2,3 and 4 are needed. These assumptions play an important role in proving theorem 1 which will be given later.

Assumption 2: The pairs $\left(A_{j j}, C_{j j}\right), \quad j=1,2$, are detectable.

Assumption 3: The Hamiltonian matrices $Z_{j j}, j=1,2$, have no eigenvalues on the imaginary axis, where

$$
Z_{j j}:=\left[\begin{array}{cc}
A_{j j}^{\mathrm{T}} & -S_{j j} \\
-U_{j j} & -A_{j j}
\end{array}\right] .
$$




\section{Assumption 4:}

$\operatorname{rank}\left[\begin{array}{ccccc}s I_{n_{0}}-A_{00}^{\mathrm{T}} & -A_{10}^{\mathrm{T}} & -A_{20}^{\mathrm{T}} & C_{10}^{\mathrm{T}} & C_{20}^{\mathrm{T}} \\ -A_{01}^{\mathrm{T}} & -A_{11}^{\mathrm{T}} & 0 & C_{11}^{\mathrm{T}} & 0 \\ -A_{02}^{\mathrm{T}} & 0 & -A_{22}^{\mathrm{T}} & 0 & C_{22}^{\mathrm{T}}\end{array}\right]=\bar{n}$,

$\operatorname{rank}\left[\begin{array}{ccccc}s I_{n_{0}}-A_{00} & -A_{01} & -A_{02} & D_{01} & D_{02} \\ -A_{10} & -A_{11} & 0 & D_{11} & 0 \\ -A_{20} & 0 & -A_{22} & 0 & D_{22}\end{array}\right]=\bar{n},($

with $\forall s \in \mathbf{C}, \operatorname{Re}[s] \geqslant 0$ and $\bar{n}:=n_{1}+n_{2}$.

Let $\bar{Y}_{00}, \bar{Y}_{10}, \bar{Y}_{20}, \bar{Y}_{11}, \bar{Y}_{21}$ and $\bar{Y}_{22}$ be the limiting solutions of the GMARE (9a) as $\varepsilon_{j} \rightarrow 0^{+}, j=1,2$. In this case the following zeroth-order equations are obtained by partitioning the GMARE (9a):

$$
\begin{aligned}
& A_{s} \bar{Y}_{00}+\bar{Y}_{00} A_{s}^{\mathrm{T}}-\bar{Y}_{00} S_{s} \bar{Y}_{00}+U_{s}=0, \\
& \bar{Y}_{j 0}^{\mathrm{T}}=\left[\begin{array}{ll}
-\bar{Y}_{00} & I_{n_{0}}
\end{array}\right] Z_{0 j} Z_{j j}^{-1}\left[\begin{array}{c}
I_{n_{j}} \\
\bar{Y}_{j j}
\end{array}\right], \\
& A_{j j} \bar{Y}_{j j}+\bar{Y}_{j j} A_{j j}^{\mathrm{T}}-\bar{Y}_{j j} S_{j j} \bar{Y}_{j j}+U_{j j}=0,
\end{aligned}
$$

where

$$
\begin{aligned}
Z_{s} & :=Z_{00}-Z_{01} Z_{11}^{-1} Z_{10}-Z_{02} Z_{22}^{-1} Z_{20}=\left[\begin{array}{cc}
A_{s}^{\mathrm{T}} & -S_{s} \\
-U_{s} & -A_{s}
\end{array}\right], \\
Z_{00} & :=\left[\begin{array}{cc}
A_{00}^{\mathrm{T}} & -S_{00} \\
-U_{00} & -A_{00}
\end{array}\right], \quad Z_{0 j}:=\left[\begin{array}{cc}
A_{j 0}^{\mathrm{T}} & -S_{0 j} \\
-U_{0 j} & -A_{0 j}
\end{array}\right], \\
Z_{j 0} & :=\left[\begin{array}{cc}
A_{0 j}^{\mathrm{T}} & -S_{0 j}^{\mathrm{T}} \\
-U_{0 j}^{\mathrm{T}} & -A_{j 0}
\end{array}\right], \quad j=1,2 .
\end{aligned}
$$

It should be noted that, if (11c) has a stabilizing solution, then the Hamiltonian matrices $Z_{j j}$ have not eigenvalues on the imaginary axis and therefore these matrices are non-singular (Zhou 1998).

In the rest of this section, relations between the GMARE (9a) and the zeroth-order equations (11) are considered. Before doing that, useful results for the ARE (11a) are given.

Lemma 1: Under assumptions 1-4, there exists a matrix $\mathcal{C} \in \mathbf{R}^{\bar{p} \times n_{0}}, \bar{p}:=p_{1}+p_{2}$ and a matrix $\mathcal{D} \in \mathbf{R}^{n_{0} \times \bar{q}}, \bar{q}:=$ $q_{1}+q_{2} \quad$ such that $S_{s}=\mathcal{C}^{\mathrm{T}} V^{-1} \mathcal{C}, \quad U_{s}=\mathcal{D} W \mathcal{D}^{\mathrm{T}}$. Moreover, the triple $\left(A_{s}, \mathcal{C}, \mathcal{D}\right)$ is stabilizable and detectable.

Proof: Since the proof of lemma 1 is easy by a similar argument to that of Mukaidani et al. (2002d), it is omitted.
The limiting behaviour of $Y_{e}$ as the parameter $\|\mu\|:=\left(\varepsilon_{1}^{2}+\varepsilon_{2}^{2}\right)^{1 / 2} \rightarrow 0^{+}$is described by the following theorem.

Theorem 1: Under Assumptions 1-4, there exists a small $\sigma^{*}$ such that for all $\|\mu\| \in\left(0, \sigma^{*}\right)$ the MARE (8) admits a symmetric positive semidefinite stabilizing solution $Y_{e}$ which can be written as

$$
Y_{e}=\left[\begin{array}{ccc}
\bar{Y}_{00}+O(\|\mu\|) & \bar{Y}_{10}^{\mathrm{T}}+O(\|\mu\|) & \bar{Y}_{20}^{\mathrm{T}}+O(\|\mu\|) \\
\bar{Y}_{10}+O(\|\mu\|) & \varepsilon_{1}^{-1}\left(\bar{Y}_{11}+O(\|\mu\|)\right) & \left(\varepsilon_{1} \varepsilon_{2}\right)^{-1 / 2} O(\|\mu\|) \\
\bar{Y}_{20}+O(\|\mu\|) & \left(\varepsilon_{1} \varepsilon_{2}\right)^{-1 / 2} O(\|\mu\|) & \varepsilon_{2}^{-1}\left(\bar{Y}_{22}+O(\|\mu\|)\right)
\end{array}\right],
$$

where $\mu:=\left[\begin{array}{ll}\varepsilon_{1} & \varepsilon_{2}\end{array}\right]$.

Proof: The proof of this theorem is acheived by using the implicit function theorem (Gajić 1988, Gajić et al. 1998) to (9a). To do this, it is enough to show that the corresponding Jacobian is non-singular at $\varepsilon_{j}=0$, $j=1,2$. It can be shown, after some algebra, that the Jacobian of (9a) in the limit as $\mu \rightarrow \bar{\mu}=\left[\begin{array}{ll}0 & 0\end{array}\right]$ is given by

$$
\mathbf{J}=\left[\begin{array}{cccccc}
J_{00} & J_{01} & J_{02} & 0 & 0 & 0 \\
J_{10} & J_{11} & 0 & J_{13} & J_{14} & 0 \\
J_{20} & 0 & J_{22} & 0 & J_{24} & J_{25} \\
0 & 0 & 0 & J_{33} & 0 & 0 \\
0 & 0 & 0 & 0 & J_{44} & 0 \\
0 & 0 & 0 & 0 & 0 & J_{55}
\end{array}\right],
$$

and

$$
\begin{aligned}
J_{00} & =I_{n_{0}} \otimes H_{00}+H_{00} \otimes I_{n_{0}}, \\
J_{0 j} & =\left(I_{n_{0}} \otimes H_{0 j}\right) U_{n_{0} n_{j}}+H_{0 j} \otimes I_{n_{0}}, \\
J_{j 0} & =H_{j 0} \otimes I_{n_{0}}, \quad J_{j j}=H_{j j} \otimes I_{n_{0}}, \\
J_{13} & =I_{n_{1}} \otimes H_{01}, \quad J_{14}=\bar{\alpha}^{1 / 2}\left(I_{n_{1}} \otimes H_{02}\right) U_{n_{1} n_{2}}, \\
J_{24} & =\frac{1}{\bar{\alpha}^{1 / 2}}\left(I_{n_{2}} \otimes H_{01}\right), \quad J_{25}=I_{n_{2}} \otimes H_{02}, \\
J_{33} & =I_{n_{1}} \otimes H_{11}+H_{11} \otimes I_{n_{1}}, \\
J_{44} & =\bar{\alpha}^{1 / 2} H_{22} \otimes I_{n_{1}}+\frac{1}{\bar{\alpha}^{1 / 2}} I_{n_{2}} \otimes H_{11}, \\
J_{55} & =I_{n_{2}} \otimes H_{22}+H_{22} \otimes I_{n_{2}}, \\
H_{00} & =A_{00}-\bar{Y}_{00} S_{00}-\bar{Y}_{10}^{\mathrm{T}} S_{01}^{\mathrm{T}}-\bar{Y}_{20}^{\mathrm{T}} S_{02}^{\mathrm{T}}, \\
H_{j 0} & =A_{j 0}-\bar{Y}_{j j} S_{0 j}^{\mathrm{T}}, \quad H_{0 j}=A_{0 j}-\bar{Y}_{00} S_{0 j}-\bar{Y}_{j 0}^{\mathrm{T}} S_{j j}, \\
H_{j j} & =A_{j j}-\bar{Y}_{j j} S_{j j}, \quad j=1,2 .
\end{aligned}
$$

The Jacobian (13) can be expressed as

$\operatorname{det} \mathbf{J}=\operatorname{det} J_{11} \operatorname{det} J_{22} \operatorname{det} J_{33} \operatorname{det} J_{44} \operatorname{det} J_{55}$

$$
\times \operatorname{det}\left[I_{n_{0}} \otimes H_{0}+H_{0} \otimes I_{n_{0}}\right],
$$


where $H_{0} \equiv H_{00}-H_{01} H_{11}^{-1} H_{10}-H_{02} H_{22}^{-1} H_{20}$. Obviously, $J_{i j}, j=1, \ldots, 5$ are non-singular because the matrices $H_{j j}=A_{j j}-\bar{Y}_{j j} S_{j j}, j=1,2$, are stable. After some straightforward but tedious algebra, it can be easily seen that $A_{s}-\bar{Y}_{00} S_{s}=H_{00}-H_{01} H_{11}^{-1} H_{10}-$ $H_{02} H_{22}^{-1} H_{20}=H_{0}$. Therefore, the matrix $H_{0}$ is stable if the ARE (11a) has a positive semidefinite stabilizing solution. Thus, det $\mathbf{J} \neq 0$. The conclusion of Theorem 1 is obtained directly by using the implicit function theorem. The remainder of the proof is to show that $Y_{e}$ is a positive semidefinite stabilizing solution. However, the proof is omitted since it is similar to that given by Mukaidani et al. (2002d).

\section{The recursive algorithm}

Now, let us define $\mathcal{E}:=\left(\varepsilon_{1} \varepsilon_{2}\right)^{1 / 2}$. By making use of the zeroth-order solutions (11), the solution (12) can be changed as follows:

$$
Y_{p q}=\bar{Y}_{p q}+\mathcal{E} M_{p q}, \quad p q=00,10,20,11,21,22,
$$

where $\quad M_{00}=M_{00}^{\mathrm{T}}, \quad M_{11}=M_{11}^{\mathrm{T}}, \quad M_{22}=M_{22}^{\mathrm{T}}, \quad$ and $\bar{Y}_{21}=0$.

Substituting (15) into (9a) and subtracting (11) from (9a), the following error equations (16) are given:

$$
\begin{aligned}
& H_{00} M_{00}+M_{00} H_{00}^{\mathrm{T}}+H_{01} M_{10}+M_{10}^{\mathrm{T}} H_{01}^{\mathrm{T}}+H_{02} M_{20} \\
& +M_{20}^{\mathrm{T}} H_{02}^{\mathrm{T}}-\mathcal{E}\left(M_{00} S_{00} M_{00}+M_{10}^{\mathrm{T}} S_{01}^{\mathrm{T}} M_{00}\right. \\
& +M_{00} S_{01} M_{10}+M_{20}^{\mathrm{T}} S_{02}^{\mathrm{T}} M_{00}+M_{00} S_{02} M_{20} \\
& \left.+M_{10}^{\mathrm{T}} S_{11} M_{10}+M_{20}^{\mathrm{T}} S_{22} M_{20}\right)=0 \\
& M_{00} H_{10}^{\mathrm{T}}+M_{10}^{\mathrm{T}} H_{11}+H_{01} M_{11}+\alpha^{1 / 2} H_{02} M_{21} \\
& +\frac{\varepsilon_{1}}{\mathcal{E}} H_{00} Y_{10}^{\mathrm{T}}-\mathcal{E}\left(M_{00} S_{01} M_{11}+M_{10}^{\mathrm{T}} S_{11} M_{11}\right) \\
& -\mathcal{E}^{1 / 2}\left(M_{00} S_{02} M_{21}+M_{20}^{\mathrm{T}} S_{22} M_{21}\right) \\
& -\varepsilon_{1}\left(M_{00} S_{00}+M_{10}^{\mathrm{T}} S_{01}^{\mathrm{T}}+M_{20}^{\mathrm{T}} S_{02}^{\mathrm{T}}\right) Y_{10}^{\mathrm{T}}=0, \\
& M_{00} H_{20}^{\mathrm{T}}+M_{20}^{\mathrm{T}} H_{22}+H_{02} M_{22}+\frac{1}{\alpha^{1 / 2}} H_{01} M_{21}^{\mathrm{T}} \\
& +\frac{\varepsilon_{2}}{\mathcal{E}} H_{00} Y_{20}^{\mathrm{T}}-\mathcal{E}\left(M_{00} S_{02} M_{22}+M_{20}^{\mathrm{T}} S_{22} M_{22}\right) \\
& \quad-\frac{\mathcal{E}}{\alpha^{1 / 2}}\left(M_{00} S_{01} M_{21}^{\mathrm{T}}+M_{10}^{\mathrm{T}} S_{11}^{\mathrm{T}} M_{21}^{\mathrm{T}}\right) \\
& \quad-\varepsilon_{2}\left(M_{00} S_{00}+M_{10}^{\mathrm{T}} S_{01}^{\mathrm{T}}+M_{20}^{\mathrm{T}} S_{02}^{\mathrm{T}}\right) Y_{20}^{\mathrm{T}}=0,
\end{aligned}
$$

$$
\begin{aligned}
& H_{11} M_{11}+M_{11} H_{11}^{\mathrm{T}}+\frac{\varepsilon_{1}}{\mathcal{E}}\left(H_{10} \bar{Y}_{10}^{\mathrm{T}}+\bar{Y}_{10} H_{10}^{\mathrm{T}}\right) \\
& +\varepsilon_{1}\left(H_{10} M_{10}^{\mathrm{T}}+M_{10} H_{10}^{\mathrm{T}}\right)-\frac{\varepsilon_{1}^{2}}{\mathcal{E}} Y_{10} S_{00} Y_{10}^{\mathrm{T}} \\
& -\varepsilon_{1}\left(M_{11} S_{01}^{\mathrm{T}} Y_{10}^{\mathrm{T}}+Y_{10} S_{01} M_{11}\right) \\
& -\varepsilon_{1} \alpha^{1 / 2}\left(M_{21}^{\mathrm{T}} S_{02}^{\mathrm{T}} Y_{10}^{\mathrm{T}}+Y_{10} S_{02} M_{21}\right) \\
& -\mathcal{E}\left(M_{11} S_{11} M_{11}+\alpha M_{21}^{\mathrm{T}} S_{22} M_{21}\right)=0, \\
& \alpha^{1 / 2} M_{21}^{\mathrm{T}} H_{22}+\frac{1}{\alpha^{1 / 2}} H_{11} M_{21}^{\mathrm{T}}+\frac{\varepsilon_{1}}{\mathcal{E}} \bar{Y}_{10} H_{20}^{\mathrm{T}}+\frac{\varepsilon_{2}}{\mathcal{E}} H_{10} \bar{Y}_{20}^{\mathrm{T}} \\
& +\varepsilon_{1} M_{10} H_{20}^{\mathrm{T}}+\varepsilon_{2} H_{10} M_{20}^{\mathrm{T}} \\
& -\varepsilon_{1}\left(Y_{10} S_{02} M_{22}+\frac{1}{\alpha^{1 / 2}} Y_{10} S_{01} M_{21}^{\mathrm{T}}\right) \\
& -\varepsilon_{2}\left(M_{11} S_{01}^{\mathrm{T}} Y_{20}^{\mathrm{T}}+\alpha^{1 / 2} M_{21}^{\mathrm{T}} S_{02}^{\mathrm{T}} Y_{20}^{\mathrm{T}}\right)-\frac{\varepsilon_{1} \varepsilon_{2}}{\mathcal{E}} Y_{10} S_{00} Y_{20}^{\mathrm{T}} \\
& -\mathcal{E}\left(\alpha^{1 / 2} M_{21}^{\mathrm{T}} S_{22} M_{22}+\frac{1}{\alpha^{1 / 2}} M_{11} S_{11}^{\mathrm{T}} M_{21}^{\mathrm{T}}\right)=0, \\
& H_{22} M_{22}+M_{22} H_{22}^{\mathrm{T}}+\frac{\varepsilon_{2}}{\mathcal{E}}\left(H_{20} \bar{Y}_{20}^{\mathrm{T}}+\bar{Y}_{20} H_{20}^{\mathrm{T}}\right) \\
& +\varepsilon_{2}\left(H_{20} M_{20}^{\mathrm{T}}+M_{20} H_{20}^{\mathrm{T}}\right)-\frac{\varepsilon_{2}^{2}}{\mathcal{E}} Y_{20} S_{00} Y_{20}^{\mathrm{T}} \\
& -\varepsilon_{2}\left(M_{22} S_{02}^{\mathrm{T}} Y_{20}^{\mathrm{T}}+Y_{20} S_{02} M_{22}\right) \\
& -\frac{\varepsilon_{2}}{\alpha^{1 / 2}}\left(M_{21} S_{01}^{\mathrm{T}} Y_{20}^{\mathrm{T}}+Y_{20} S_{01} M_{21}^{\mathrm{T}}\right) \\
& -\mathcal{E}\left(M_{22} S_{22} M_{22}+\frac{1}{\alpha} M_{21} S_{11} M_{21}^{\mathrm{T}}\right)=0 .
\end{aligned}
$$

Hence, the following iterative algorithm which is based on the recursive algorithm is given:

$$
\begin{aligned}
H_{11} & M_{11}^{(i+1)}+M_{11}^{(i+1)} H_{11}^{\mathrm{T}} \\
= & -\frac{\varepsilon_{1}}{\mathcal{E}}\left(H_{10} \bar{Y}_{10}^{\mathrm{T}}+\bar{Y}_{10} H_{10}^{\mathrm{T}}\right)-\varepsilon_{1}\left(H_{10} M_{10}^{(i) \mathrm{T}}+M_{10}^{(i)} H_{10}^{\mathrm{T}}\right) \\
& +\frac{\varepsilon_{1}^{2}}{\mathcal{E}} Y_{10}^{(i)} S_{00} Y_{10}^{(i) \mathrm{T}}+\varepsilon_{1}\left(M_{11}^{(i)} S_{01}^{\mathrm{T}} Y_{10}^{(i) \mathrm{T}}+Y_{10}^{(i)} S_{01} M_{11}^{(i)}\right) \\
& +\varepsilon_{1} \alpha^{1 / 2}\left(M_{21}^{(i) \mathrm{T}} S_{02}^{\mathrm{T}} Y_{10}^{(i) \mathrm{T}}+Y_{10}^{(i)} S_{02} M_{21}^{(i)}\right) \\
& +\mathcal{E}\left(M_{11}^{(i)} S_{11} M_{11}^{(i)}+\alpha M_{21}^{(i) \mathrm{T}} S_{22} M_{21}^{(i)}\right), \\
H_{22} & M_{22}^{(i+1)}+M_{22}^{(i+1)} H_{22}^{\mathrm{T}} \\
= & -\frac{\varepsilon_{2}}{\mathcal{E}}\left(H_{20} \bar{Y}_{20}^{\mathrm{T}}+\bar{Y}_{20} H_{20}^{\mathrm{T}}\right)-\varepsilon_{2}\left(H_{20} M_{20}^{(i) \mathrm{T}}+M_{20}^{(i)} H_{20}^{\mathrm{T}}\right) \\
& +\frac{\varepsilon_{2}^{2}}{\mathcal{E}} Y_{20}^{(i)} S_{00} Y_{20}^{(i) \mathrm{T}}+\varepsilon_{2}\left(M_{22}^{(i)} S_{02}^{\mathrm{T}} Y_{20}^{(i) \mathrm{T}}+Y_{20}^{(i)} S_{02} M_{22}^{(i)}\right) \\
& +\frac{\varepsilon_{2}}{\alpha^{1 / 2}}\left(M_{21}^{(i)} S_{01}^{\mathrm{T}} Y_{20}^{(i) \mathrm{T}}+Y_{20}^{(i)} S_{01} M_{21}^{(i) \mathrm{T}}\right) \\
& +\mathcal{E}\left(M_{22}^{(i)} S_{22} M_{22}^{(i)}+\frac{1}{\alpha} M_{21}^{(i)} S_{11} M_{21}^{(i) \mathrm{T}}\right),
\end{aligned}
$$




$$
\begin{aligned}
& \alpha^{1 / 2} M_{21}^{(i+1) T} H_{22}+\frac{1}{\alpha^{1 / 2}} H_{11} M_{21}^{(i+1) T} \\
&=-\frac{\varepsilon_{1}}{\mathcal{E}} \bar{Y}_{10} H_{20}^{\mathrm{T}}-\frac{\varepsilon_{2}}{\mathcal{E}} H_{10} \bar{Y}_{20}^{\mathrm{T}}-\varepsilon_{1} M_{10}^{(i)} H_{20}^{\mathrm{T}}-\varepsilon_{2} H_{10} M_{20}^{(i) \mathrm{T}} \\
&+\frac{\varepsilon_{1} \varepsilon_{2}}{\mathcal{E}} Y_{10}^{(i)} S_{00} Y_{20}^{(i) \mathrm{T}} \\
&+\varepsilon_{1}\left(Y_{10}^{(i)} S_{02} M_{22}^{(i)}+\frac{1}{\alpha^{1 / 2}} Y_{10}^{(i)} S_{01} M_{21}^{(i) \mathrm{T}}\right) \\
&+\varepsilon_{2}\left(M_{11}^{(i)} S_{01}^{\mathrm{T}} Y_{20}^{(i) \mathrm{T}}+\alpha^{1 / 2} M_{21}^{(i) \mathrm{T}} S_{02}^{\mathrm{T}} Y_{20}^{(i) \mathrm{T}}\right) \\
&+\mathcal{E}\left(\alpha^{1 / 2} M_{21}^{(i) \mathrm{T}} S_{22} M_{22}^{(i)}+\frac{1}{\alpha^{1 / 2}} M_{11}^{(i)} S_{11}^{\mathrm{T}} M_{21}^{(i) \mathrm{T}}\right) \\
& H_{0} M_{00}^{(i+1)}+M_{00}^{(i+1)} H_{0}^{\mathrm{T}} \\
&=-H_{01} H_{11}^{-1} L_{01}^{(i) \mathrm{T}}-L_{01}^{(i)} H_{11}^{-\mathrm{T}} H_{01}^{\mathrm{T}}-H_{02} H_{22}^{-1} L_{02}^{(i) \mathrm{T}} \\
&-L_{02}^{(i)} H_{22}^{-\mathrm{T}} H_{02}^{\mathrm{T}}+\mathcal{E}\left(M_{00}^{(i)} S_{00} M_{00}^{(i)}+M_{10}^{(i) \mathrm{T}} S_{01}^{\mathrm{T}} M_{00}^{(i)}\right. \\
&+M_{00}^{(i)} S_{01} M_{10}^{(i)}+M_{20}^{(i) \mathrm{T}} S_{02}^{\mathrm{T}} M_{00}^{(i)}+M_{00}^{(i)} S_{02} M_{20}^{(i)} \\
&\left.+M_{10}^{(i) \mathrm{T}} S_{11}^{\mathrm{T}} M_{10}^{(i)}+M_{20}^{(i) \mathrm{T}} S_{22} M_{20}^{(i)}\right) \\
& M_{j 0}^{(i+1) \mathrm{T}}=\left(L_{0 j}^{(i)}-M_{00}^{(i+1)} H_{j 0}^{\mathrm{T}}\right) H_{j j}^{-1}
\end{aligned}
$$

where $j=1,2$,

$$
\begin{aligned}
L_{01}^{(i)}= & -H_{01} M_{11}^{(i+1)}-\alpha^{1 / 2} H_{02} M_{21}^{(i+1)}-\frac{\varepsilon_{1}}{\mathcal{E}} H_{00}^{\mathrm{T}} Y_{10}^{\mathrm{T}} \\
& +\mathcal{E}\left(M_{00}^{(i)} S_{01} M_{11}^{(i)}+M_{10}^{(i) \mathrm{T}} S_{11} M_{11}^{(i)}\right) \\
& +\mathcal{E} \alpha^{1 / 2}\left(M_{00}^{(i)} S_{02} M_{21}^{(i)}+M_{20}^{(i) \mathrm{T}} S_{22}^{\mathrm{T}} M_{21}^{(i)}\right) \\
& +\varepsilon_{1}\left(M_{00}^{(i)} S_{00}+M_{10}^{(i) \mathrm{T}} S_{01}^{\mathrm{T}}+M_{20}^{(i) \mathrm{T}} S_{02}^{\mathrm{T}}\right) Y_{10}^{(i) \mathrm{T}} \\
L_{02}^{(i)}= & -H_{02} M_{22}^{(i+1)}-\frac{1}{\alpha^{1 / 2}} H_{01}^{\mathrm{T}} M_{21}^{(i+1) T}-\frac{\varepsilon_{2}}{\mathcal{E}} H_{00}^{\mathrm{T}} Y_{20}^{\mathrm{T}} \\
& +\mathcal{E}\left(M_{00}^{(i)} S_{02} M_{22}^{(i)}+M_{20}^{(i) \mathrm{T}} S_{22} M_{22}^{(i)}\right) \\
& +\frac{\mathcal{E}}{\alpha^{1 / 2}}\left(M_{00}^{(i)} S_{01} M_{21}^{(i) \mathrm{T}}+M_{10}^{(i) \mathrm{T}} S_{11}^{\mathrm{T}} M_{21}^{(i) \mathrm{T}}\right) \\
& +\varepsilon_{2}\left(M_{00}^{(i)} S_{00}+M_{10}^{(i) \mathrm{T}} S_{01}^{\mathrm{T}}+M_{20}^{(i) \mathrm{T}} S_{02}^{\mathrm{T}}\right) Y_{20}^{(i) \mathrm{T}}, \\
Y_{j 0}^{(i)}= & \bar{Y}_{j 0}+\mathcal{E} M_{j 0}^{(i)}, \quad i=0,1, \ldots, \\
M_{00}^{(0)}= & M_{10}^{(0)}=M_{20}^{(0)}=M_{11}^{(0)}=M_{21}^{(0)}=M_{22}^{(0)}=0 .
\end{aligned}
$$

The following theorem indicates the convergence of the algorithm (17).

Theorem 2: Under assumptions 1-4, there exist unique and bounded solutions $M_{p q}$ of the error equation in a neighbourhood of $\|\mu\|=0$. Moreover, the algorithm (17) converges to the exact solutions $M_{p q}$ with the rate of the convergence of $O\left(\|\mu\|^{i}\right)$, that is

$$
\left\|M_{p q}-M_{p q}^{(i)}\right\|=O\left(\|\mu\|^{i}\right), \quad i=1,2, \ldots .
$$

Proof: As a starting point it must be shown that there exist the unique and bounded solutions of $M_{p q}$ in a neighbourhood of $\|\mu\|=0$. To prove that by the implicit function theorem, it is enough to show that the corresponding Jacobian $\mathbf{J}_{0}$ of (16) is non-singular at $\|\mu\|=0$. The Jacobian is given by

$$
\mathbf{J}_{0}=\mathbf{J}
$$

Taking into consideration the fact that $\mathbf{J}$ is non-singular at $\|\mu\|=0, \mathbf{J}_{0}$ is also non-singular. Therefore, there exist unique and bounded solutions of the error equations (16).

Secondly, the proof of (18) uses mathematical induction. Subtracting (17) from (16), the following equations are derived:

$$
\begin{aligned}
& H_{00}\left(M_{00}-M_{00}^{(i+1)}\right)+\left(M_{00}-M_{00}^{(i+1)}\right) H_{00}^{\mathrm{T}} \\
&+H_{01}\left(M_{10}-M_{10}^{(i+1)}\right)+\left(M_{10}-M_{10}^{(i+1)}\right)^{\mathrm{T}} H_{01}^{\mathrm{T}} \\
&+H_{02}\left(M_{20}-M_{20}^{(i+1)}\right)+\left(M_{20}-M_{20}^{(i+1)}\right)^{\mathrm{T}} H_{02}^{\mathrm{T}} \\
&= \mathcal{E} \mathcal{F}_{1}\left(M_{00}^{(i)}, M_{10}^{(i)}, M_{20}^{(i)}, \varepsilon_{1}, \varepsilon_{2}\right), \\
&(\left.M_{00}-M_{00}^{(i+1)}\right) H_{10}^{\mathrm{T}}+\left(M_{10}-M_{10}^{(i+1)}\right)^{\mathrm{T}} H_{11} \\
&+H_{01}\left(M_{11}-M_{11}^{(i+1)}\right)+\alpha^{1 / 2} H_{02}\left(M_{21}-M_{21}^{(i+1)}\right) \\
&= \mathcal{E} \mathcal{F}_{2}\left(M_{00}^{(i)}, M_{10}^{(i)}, M_{20}^{(i)}, M_{11}^{(i)}, M_{21}^{(i)}, \varepsilon_{1}, \varepsilon_{2}\right), \\
&\left(M_{00}-M_{00}^{(i+1)}\right) H_{20}^{\mathrm{T}}+\left(M_{20}-M_{20}^{(i+1)}\right)^{\mathrm{T}} H_{22} \\
& \quad+H_{02}\left(M_{22}-M_{22}^{(i+1)}\right)+\frac{1}{\alpha^{1 / 2}} H_{01}\left(M_{21}-M_{21}^{(i+1)}\right)^{\mathrm{T}} \\
&=\mathcal{E F}_{3}\left(M_{00}^{(i)}, M_{10}^{(i)}, M_{20}^{(i)}, M_{21}^{(i)}, M_{22}^{(i)}, \varepsilon_{1}, \varepsilon_{2}\right), \\
& H_{11}\left(M_{11}-M_{11}^{(i+1)}\right)+\left(M_{11}-M_{11}^{(i+1)}\right) H_{11}^{\mathrm{T}} \\
&=\mathcal{E F}_{4}\left(M_{10}^{(i)}, M_{11}^{(i)}, M_{21}^{(i)}, \varepsilon_{1}, \varepsilon_{2}\right), \\
& \alpha^{1 / 2}\left(M_{21}-M_{21}^{(i+1)}\right)^{\mathrm{T}} H_{22}+\frac{1}{\alpha^{1 / 2}} H_{11}\left(M_{21}-M_{21}^{(i+1)}\right)^{\mathrm{T}} \\
&= \mathcal{E} \mathcal{F}_{5}\left(M_{10}^{(i)}, M_{20}^{(i)}, M_{11}^{(i)}, M_{21}^{(i)}, M_{22}^{(i)}, \varepsilon_{1}, \varepsilon_{2}\right), \\
& H_{22}\left(M_{22}-M_{22}^{(i+1)}\right)+\left(M_{22}-M_{22}^{(i+1)}\right) H_{22}^{\mathrm{T}} \\
&=\mathcal{E \mathcal { F }}_{6}\left(M_{20}^{(i)}, M_{21}^{(i)}, M_{22}^{(i)}, \varepsilon_{1}, \varepsilon_{2}\right), \\
& \quad
\end{aligned}
$$

where $\mathcal{F}_{j}, j=1, \ldots, 6$, are appropriate implicit functions of the matrices $M_{p q}^{(i)}$. When $i=0$ for the equations (20), using the standard properties of the algebraic Lyapunov equation (ALE) (Zhou 1998), and taking into consideration the stable matrices $H_{11}$ and $H_{22}$ it follows that

$$
\begin{aligned}
& H_{j j}\left(M_{j j}-M_{j j}^{(1)}\right)+\left(M_{j j}-M_{j j}^{(1)}\right) H_{j j}^{\mathrm{T}}=O(\|\mu\|) \\
& \Rightarrow M_{j j}-M_{j j}^{(1)}=O(\|\mu\|), \quad j=1,2, \\
& \alpha^{1 / 2}\left(M_{21}-M_{21}^{(1)}\right)^{\mathrm{T}} H_{22}+\frac{1}{\alpha^{1 / 2}} H_{11}\left(M_{21}-M_{21}^{(1)}\right)^{\mathrm{T}}=O(\|\mu\|) \\
& \Rightarrow M_{21}-M_{21}^{(1)}=O(\|\mu\|) .
\end{aligned}
$$


Since $H_{11}$ and $H_{22}$ are stable, the following ALE holds:

$$
\begin{aligned}
& H_{0}\left(M_{00}-M_{00}^{(1)}\right)+\left(M_{00}-M_{00}^{(1)}\right) H_{0}^{\mathrm{T}}=O(\|\mu\|), \\
& \left(M_{j 0}-M_{j 0}^{(1)}\right)^{\mathrm{T}}=-\left(M_{00}-M_{00}^{(1)}\right) H_{j 0}^{\mathrm{T}} H_{j j}^{-1}+O(\|\mu\|), \quad j=1,2 .
\end{aligned}
$$

Using the above similar technique, and by stability of $H_{0}$, the following relation holds:

$$
\left\|M_{p q}-M_{p q}^{(1)}\right\|=O(\|\mu\|), \quad p q=00,10,20,11,21,22 .
$$

When $i=k(i \geq 2)$, it is assumed that $\left\|M_{p q}-M_{p q}^{(k)}\right\|=$ $O\left(\|\mu\|^{k}\right)$. Using this assumption, the right-hand side of the equation (20a) can be changed as follows:

$$
\begin{aligned}
& \mathcal{E F}_{1}\left(M_{00}^{(k)}, M_{10}^{(k)}, M_{20}^{(k)}, \varepsilon_{1}, \varepsilon_{2}\right) \\
&= \mathcal{E}\left(M_{00} S_{00} M_{00}+M_{10}^{\mathrm{T}} S_{01}^{\mathrm{T}} M_{00}+M_{00} S_{01} M_{10}\right. \\
&+M_{20}^{\mathrm{T}} S_{02}^{\mathrm{T}} M_{00}+M_{00} S_{02} M_{20}+M_{10}^{\mathrm{T}} S_{11} M_{10} \\
&\left.+M_{20}^{\mathrm{T}} S_{22} M_{20}\right)-\mathcal{E}\left(M_{00}^{(k)} S_{00} M_{00}^{(k)}+M_{10}^{(k) T} S_{01}^{\mathrm{T}} M_{00}^{(k)}\right. \\
&+M_{00}^{(k)} S_{01} M_{10}^{(k)}+M_{20}^{(k) \mathrm{T}} S_{02}^{\mathrm{T}} M_{00}^{(k)}+M_{00}^{(k)} S_{02} M_{20}^{(k)} \\
&\left.+M_{10}^{(k) \mathrm{T}} S_{11} M_{10}^{(k)}+M_{20}^{(k) \mathrm{T}} S_{22} M_{20}^{(k)}\right) \\
&= \mathcal{E} O\left(\|\mu\|^{k}\right)=O\left(\|\mu\|^{k+1}\right) .
\end{aligned}
$$

Similarly, the following equations are satisfied:

$$
\begin{aligned}
& H_{00}\left(M_{00}-M_{00}^{(k+1)}\right)+\left(M_{00}-M_{00}^{(k+1)}\right) H_{00}^{\mathrm{T}} \\
& \quad+H_{01}\left(M_{10}-M_{10}^{(k+1)}\right)+\left(M_{10}-M_{10}^{(k+1)}\right)^{\mathrm{T}} H_{01}^{\mathrm{T}} \\
& \quad+H_{02}\left(M_{20}-M_{20}^{(k+1)}\right)+\left(M_{20}-M_{20}^{(k+1)}\right)^{\mathrm{T}} H_{02}^{\mathrm{T}}=O\left(\|\mu\|^{k+1}\right), \\
& \left(M_{00}-M_{00}^{(k+1)}\right) H_{10}^{\mathrm{T}}+\left(M_{10}-M_{10}^{(k+1)}\right)^{\mathrm{T}} H_{11} \\
& \quad+H_{01}\left(M_{11}-M_{11}^{(k+1)}\right)+\alpha^{1 / 2} H_{02}\left(M_{21}-M_{21}^{(k+1)}\right) \\
& =O\left(\|\mu\|^{k+1}\right), \\
& \left(M_{00}-M_{00}^{(k+1)}\right) H_{20}^{\mathrm{T}}+\left(M_{20}-M_{20}^{(k+1)}\right)^{\mathrm{T}} H_{22} \\
& +H_{02}\left(M_{22}-M_{22}^{(k+1)}\right)+\frac{1}{\alpha^{1 / 2}} H_{01}\left(M_{21}-M_{21}^{(k+1)}\right)^{\mathrm{T}} \\
& =O\left(\|\mu\|^{k+1}\right), \\
& H_{j j}\left(M_{j j}-M_{j j}^{(k+1)}\right)+\left(M_{j j}-M_{j j}^{(k+1)}\right) H_{j j}^{\mathrm{T}} \\
& =O\left(\|\mu\|^{k+1}\right), j=1,2, \\
& \alpha^{1 / 2}\left(M_{21}-M_{21}^{(k+1)}\right)^{\mathrm{T}} H_{22}+\frac{1}{\alpha^{1 / 2}} H_{11}\left(M_{21}-M_{21}^{(k+1)}\right)^{\mathrm{T}} \\
& =O\left(\|\mu\|^{k+1}\right) .
\end{aligned}
$$

By cancelling the appropriate terms, a similar proof will show that

$$
\begin{aligned}
& \left\|M_{p q}-M_{p q}^{(k+1)}\right\|=O\left(\|\mu\|^{k+1}\right), \\
& p q=00,10,20,11,21,22 .
\end{aligned}
$$

Consequently, (18) holds for all $i \in \mathbf{N}$. Thus, the proof of theorem 2 is completed.

In the paper by Mukaidani et al. (2002c), the $H_{\infty}$ optimal filtering problem for an MSPS has been studied via the recursive approach. In that paper, a MARE that has a sign indefinite quadratic term has been considered. However, the existence condition for the solution of the MARE imposes conservative conditions. Moreover, the stabilizability and detectability conditions for the reduced-order ARE were not investigated.

\section{Application}

A positive semidefinite stabilizing solution of the MARE (8) exists under assumptions 1-4. Attention is focused on the design of the high-order Kalman filters. Such a filter is obtained by using the iterative solution (17):

$$
\begin{aligned}
F_{e}^{(i)}:= & Y_{e}^{(i)} C^{\mathrm{T}} V^{-1} \\
= & {\left[\begin{array}{ccc}
\bar{Y}_{00}+\mathcal{E} M_{00}^{(i)} & \left(\bar{Y}_{10}+\mathcal{E} M_{10}^{(i)}\right)^{\mathrm{T}} & \left(\bar{Y}_{20}+\mathcal{E} M_{20}^{(i)}\right)^{\mathrm{T}} \\
\bar{Y}_{10}+\mathcal{E} M_{10}^{(i)} & \varepsilon_{1}^{-1}\left(\bar{Y}_{11}+\mathcal{E} M_{11}^{(i)}\right) & \left(\varepsilon_{1} \varepsilon_{2}\right)^{-1 / 2} \mathcal{E} M_{21}^{(i) \mathrm{T}} \\
\bar{Y}_{20}+\mathcal{E} M_{20}^{(i)} & \left(\varepsilon_{1} \varepsilon_{2}\right)^{-1 / 2} \mathcal{E} M_{21}^{(i)} & \varepsilon_{2}^{-1}\left(\bar{Y}_{22}+\mathcal{E} M_{22}^{(i)}\right)
\end{array}\right] } \\
& \times C^{\mathrm{T}} V^{-1} .
\end{aligned}
$$

Theorem 3: Under assumptions 1-4, the use of the high-order Kalman filter gain (22) results in

$$
\operatorname{Tr} W_{e}=\operatorname{Tr} Y_{e}+O\left(\|\mu\|^{2 i+1}\right),
$$

where $\operatorname{Tr} Y_{e}$ is the optimal steady-state mean square error, while $\operatorname{Tr} W_{e}$ is the near-optimal steady-state mean square error and $W_{e}$ is a positive semidefinite solution of the following multiparameter algebraic Lyapunov equation (MALE):

$$
\left(A_{e}-Y_{e}^{(i)} S\right) W_{e}+W_{e}\left(A_{e}-Y_{e}^{(i)} S\right)^{\mathrm{T}}+Y_{e}^{(i)} S Y_{e}^{(i)}+U_{e}=0 .
$$

Proof: Subtracting (8) from (24), it is easy to verify that $T_{e}=W_{e}-Y_{e}$ satisfies the following MALE:

$$
\begin{aligned}
\left(A_{e}-Y_{e}^{(i)} S\right) T_{e} & +T_{e}\left(A_{e}-Y_{e}^{(i)} S\right)_{e}^{\mathrm{T}} \\
& +\left(Y_{e}-Y_{e}^{(i)}\right) S\left(Y_{e}-Y_{e}^{(i)}\right)=0 .
\end{aligned}
$$

It is assumed that the solution $T_{e}$ of the MALE (25) has the following structure:

$$
T_{e}=\left[\begin{array}{ccc}
T_{00} & T_{10}^{\mathrm{T}} & T_{20}^{\mathrm{T}} \\
T_{10} & \varepsilon_{1}^{-1} T_{11} & \left(\varepsilon_{1} \varepsilon_{2}\right)^{-1 / 2} T_{21}^{\mathrm{T}} \\
T_{20} & \left(\varepsilon_{1} \varepsilon_{2}\right)^{-1 / 2} T_{21} & \varepsilon_{2}^{-1} T_{22}
\end{array}\right] \in \mathbf{R}^{N \times N},
$$


where $T_{00}=T_{00}^{\mathrm{T}}, T_{11}=T_{11}^{\mathrm{T}}$ and $T_{22}=T_{22}^{\mathrm{T}}$.

Using the similar technique in the work of Mukaidani et al. (2002d), the MALE (25) is equivalent to the following generalized multiparameter algebraic Lyapunov equation (GMALE) (26):

$$
\begin{aligned}
& \left(A-Y^{(i)} S\right) T^{\mathrm{T}}+T\left(A-Y^{(i)} S\right)^{\mathrm{T}} \\
& \quad+\left(Y-Y^{(i)}\right) S\left(Y-Y^{(i)}\right)^{\mathrm{T}}=0, \\
& T_{e}=T^{\mathrm{T}} \Phi_{e}^{-1}=\Phi_{e}^{-1} T .
\end{aligned}
$$

Since $Y-Y^{(i)}=O\left(\|\mu\|^{i+1}\right)$ under (18), the following GMALE holds:

$$
\left(A-Y^{(i)} S\right) T^{\mathrm{T}}+T\left(A-Y^{(i)} S\right)^{\mathrm{T}}+O\left(\|\mu\|^{2(i+1)}\right)=0 .
$$

Partitioning the GMALE (27), the following equations hold:

$$
\begin{aligned}
& \hat{H}_{00} T_{00}+T_{00} \hat{H}_{00}^{\mathrm{T}}+\hat{H}_{01} T_{10}+T_{10}^{\mathrm{T}} \hat{H}_{01}^{\mathrm{T}}+\hat{H}_{02} T_{20} \\
& \quad+T_{20}^{\mathrm{T}} \hat{H}_{02}^{\mathrm{T}}=O\left(\|\mu\|^{2(i+1)}\right) \\
& T_{00} \hat{H}_{10}^{\mathrm{T}}+T_{10}^{\mathrm{T}} \hat{H}_{11}+\hat{H}_{01} T_{11} \\
& \quad+\alpha^{1 / 2} \hat{H}_{02} T_{21}=O\left(\|\mu\|^{2(i+1)}\right), \\
& T_{00} \hat{H}_{20}^{\mathrm{T}}+T_{20}^{\mathrm{T}} \hat{H}_{22}+\hat{H}_{02} T_{22} \\
& \quad+\frac{1}{\alpha^{1 / 2}} \hat{H}_{01} T_{21}^{\mathrm{T}}=O\left(\|\mu\|^{2(i+1)}\right), \\
& \hat{H}_{j j} T_{j j}+T_{j j} \hat{H}_{j j}^{\mathrm{T}}=O\left(\|\mu\|^{2(i+1)}\right), \quad j=1,2, \\
& \alpha^{1 / 2} T_{21}^{\mathrm{T}} \hat{H}_{22}+\frac{1}{\alpha^{1 / 2}} \hat{H}_{11} T_{21}^{\mathrm{T}}=O\left(\|\mu\|^{2(i+1)}\right),
\end{aligned}
$$

where

$$
\begin{gathered}
A-Y^{(i)} S=\left[\begin{array}{ccc}
\hat{H}_{00} & \hat{H}_{01} & \hat{H}_{02} \\
\hat{H}_{10} & \hat{H}_{11} & 0 \\
\hat{H}_{20} & 0 & \hat{H}_{22}
\end{array}\right] \\
=\left[\begin{array}{ccc}
H_{00}+O(\|\mu\|) & H_{01}+O(\|\mu\|) & H_{02}+O(\|\mu\|) \\
H_{10}+O(\|\mu\|) & H_{11}+O(\|\mu\|) & O(\|\mu\|) \\
H_{20}+O(\|\mu\|) & O(\|\mu\|) & H_{22}+O(\|\mu\|)
\end{array}\right] .
\end{gathered}
$$

Since for sufficiently small $\|\mu\|, \hat{H}_{j j}=H_{j j}+O(\|\mu\|)$, $j=1,2$ is stable, it is easy to verify that $T_{j j}=$ $O\left(\|\mu\|^{2(i+1)}\right)$ and $T_{21}=O\left(\|\mu\|^{2(i+1)}\right)$ from (28d) and (28e). Moreover, using the relations $T_{j j}=O\left(\|\mu\|^{2(i+1)}\right)$ and $T_{21}=O\left(\|\mu\|^{2(i+1)}\right)$ and the non-singularlity of $\hat{H}_{j j}, j=1,2$, the following equations hold:

$\left[H_{0}+O(\|\mu\|)\right] T_{00}+T_{00}\left[H_{0}+O(\|\mu\|)\right]^{\mathrm{T}}=O\left(\|\mu\|^{2(i+1)}\right)$,

$T_{j 0}^{\mathrm{T}}=\left[T_{00} \hat{H}_{10}^{\mathrm{T}}+O\left(\|\mu\|^{2(i+1)}\right)\right] \hat{H}_{11}^{-1}, \quad j=1,2$.
Hence, $T_{00}=O\left(\|\mu\|^{2(i+1)}\right)$ and $T_{j 0}=O\left(\|\mu\|^{2(i+1)}\right)$ are satisfied. Therefore $T=O\left(\|\mu\|^{2(i+1)}\right)$, that is $T_{e}=$ $\Phi_{e}^{-1} T=O\left(\|\mu\|^{2 i+1}\right)$. Finally, $T_{e}=W_{e}-Y_{e}=O\left(\|\mu\|^{2 i+1}\right)$ results in (23).

As an extension of the well-known result of one small parameter (Kokotović et al. 1999), the following corollary is easily seen in view of theorem 3 .

Corollary 1: Under assumptions 1-4, the use of the near-optimal Kalman filter

$$
F_{e}^{(0)}=Y_{e}^{(0)} C^{\mathrm{T}} V^{-1}=\Phi_{e}^{-1}\left[\begin{array}{ccc}
\bar{Y}_{00} & \bar{Y}_{10}^{\mathrm{T}} & \bar{Y}_{20}^{\mathrm{T}} \\
\bar{Y}_{10} & \bar{Y}_{11} & 0 \\
\bar{Y}_{20} & 0 & \bar{Y}_{22}
\end{array}\right] C^{\mathrm{T}} V^{-1}
$$

means that $\operatorname{Tr} W_{e}$ satisfies

$$
\operatorname{Tr} W_{e}=\operatorname{Tr} Y_{e}+O(\|\mu\|) .
$$

Proof: Since the result of corollary 1 can be proved by using a similar technique to that for theorem 3 , the proof is omitted.

It should be noted that the result is derived by setting $i=0$ for (23). Moreover, if the parameter $\|\mu\|$ is sufficiently small, it is expected that the approximate Kalman filter (31) will be used as the optimal filter.

\section{Numerical example}

In order to demonstrate the efficiency of the proposed algorithm, a numerical example is tested. The system matrices are given by

$$
\begin{aligned}
A_{00}= & {\left[\begin{array}{ccccc}
0 & 0 & 4.5 & 0 & 1 \\
0 & 0 & 0 & 4.5 & -1 \\
0 & 0 & -0.05 & 0 & -0.1 \\
0 & 0 & 0 & -0.05 & 0.1 \\
0 & 0 & 32.7 & -32.7 & 0
\end{array}\right], } \\
A_{j j} & =\left[\begin{array}{cc}
-0.05 & 0.05 \\
0 & -0.1
\end{array}\right], \quad j=1,2, \\
A_{01}= & {\left[\begin{array}{cc}
0 & 0 \\
0 & 0 \\
0.1 & 0 \\
0 & 0 \\
0 & 0
\end{array}\right], \quad A_{02}=\left[\begin{array}{cc}
0 & 0 \\
0 & 0 \\
0 & 0 \\
0.1 & 0 \\
0 & 0
\end{array}\right], }
\end{aligned}
$$




$$
\begin{aligned}
& A_{10}=\left[\begin{array}{ccccc}
0 & 0 & 0 & 0 & 0 \\
0 & 0 & -0.4 & 0 & 0
\end{array}\right], \\
& A_{20}=\left[\begin{array}{ccccc}
0 & 0 & 0 & 0 & 0 \\
0 & 0 & 0 & -0.4 & 0
\end{array}\right], \\
& D_{01}=D_{02}=\left[\begin{array}{l}
0 \\
0 \\
0 \\
0 \\
0
\end{array}\right], \quad D_{11}=D_{22}=\left[\begin{array}{c}
0 \\
0.1
\end{array}\right] \text {, } \\
& C_{10}=\left[\begin{array}{lllll}
1 & 0 & 0 & 0 & 0 \\
0 & 1 & 0 & 0 & 0
\end{array}\right], \quad C_{20}=\left[\begin{array}{lllll}
0 & 0 & 0 & 0 & 0 \\
0 & 0 & 0 & 0 & 0
\end{array}\right], \\
& C_{11}=C_{22}=\operatorname{diag}(1,1) \text {, } \\
& V=\operatorname{diag}(0.1,0.2,0.1,0.2), \quad W=0.4 \operatorname{diag}(1,1) .
\end{aligned}
$$

The small parameters are chosen as $\varepsilon_{1}=\varepsilon_{2}=0.01$. Note that the technique proposed by Coumarbatch and Gajic (2000) for the MSPS cannot be applied because the Hamiltonian matrices $Z_{j j}, j=1,2$, have eigenvalues in common. The solution of the GMARE (9a) is
Table 1. Error $\|\mathcal{F}(Y)\|$.

\begin{tabular}{lccc}
\hline$\varepsilon_{1}$ & $\varepsilon_{2}$ & Iteration & Error \\
\hline $1 \times 10^{-2}$ & $1 \times 10^{-2}$ & 20 & $4.1659 \times 10^{-13}$ \\
$1 \times 10^{-2}$ & $5 \times 10^{-3}$ & 16 & $2.2378 \times 10^{-13}$ \\
$1 \times 10^{-3}$ & $1 \times 10^{-3}$ & 6 & $2.3606 \times 10^{-13}$ \\
$1 \times 10^{-3}$ & $5 \times 10^{-4}$ & 6 & $1.5423 \times 10^{-13}$ \\
$1 \times 10^{-4}$ & $1 \times 10^{-4}$ & 4 & $1.3164 \times 10^{-13}$ \\
$1 \times 10^{-4}$ & $5 \times 10^{-5}$ & 3 & $2.5980 \times 10^{-13}$ \\
$1 \times 10^{-5}$ & $1 \times 10^{-5}$ & 2 & $2.6053 \times 10^{-13}$ \\
\hline
\end{tabular}

It can be seen that the solution of the GMARE (9a) converge to the exact solution with accuracy of $\left\|\mathcal{F}\left(Y^{(i)}\right)\right\|<10^{-12}$ after 20 iterations. For different values of $\varepsilon_{1}$ and $\varepsilon_{2}$, in order to verify the exactitude of the solution, the errors (i.e. $\left\|\mathcal{F}\left(Y^{(i)}\right)\right\|$ ) and the necessary iteration numbers of the algorithm (17) are given in table 1. From table 1, since for sufficiently small perturbation parameters the convergence speed is quite good, the resulting algorithm of this paper is very useful.

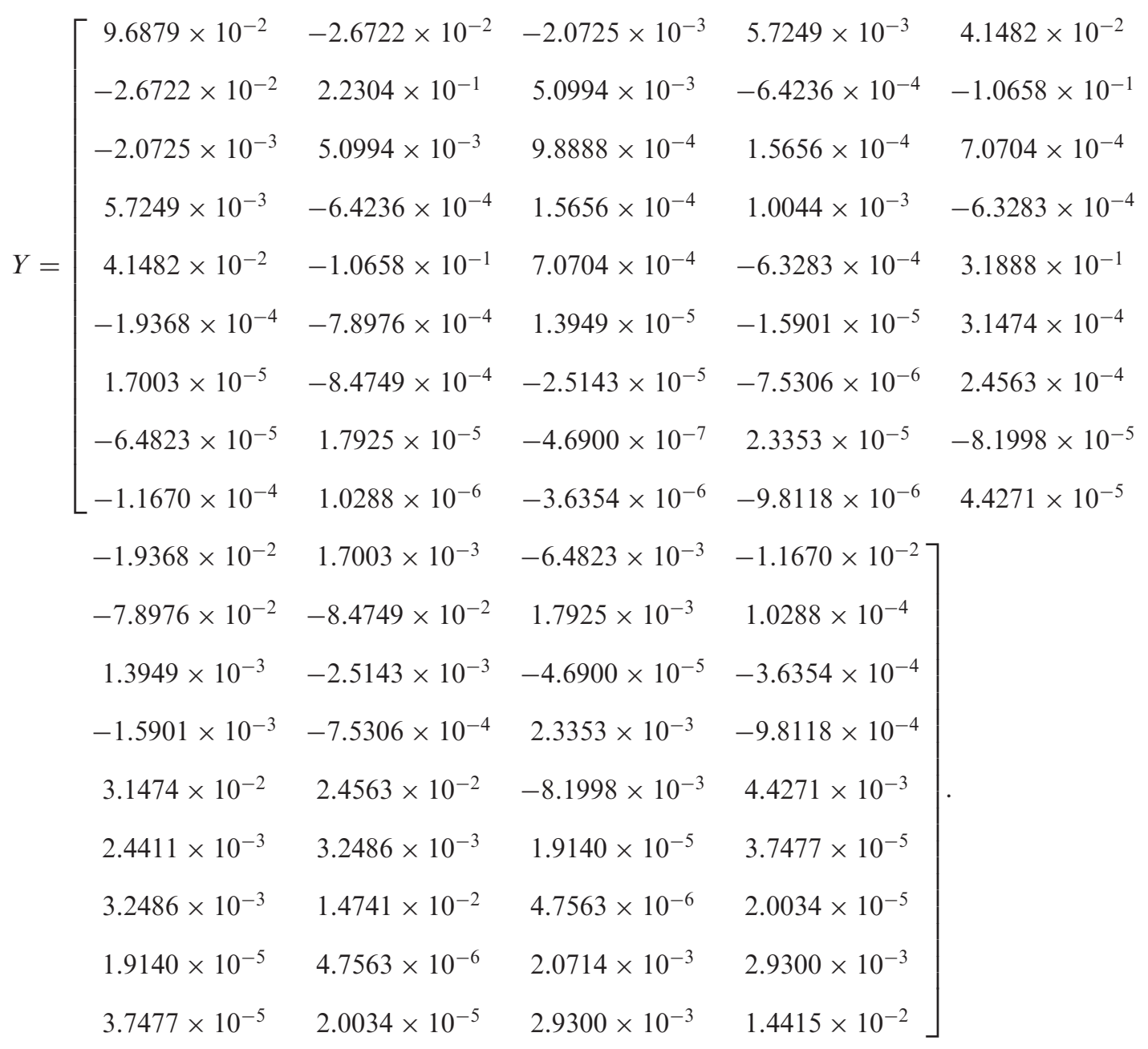


Table 2. $\quad \eta^{(i)}, i=0,1,2$.

\begin{tabular}{lcccc}
\hline$\varepsilon_{1}$ & $\varepsilon_{2}$ & $\eta^{(0)}$ & $\eta^{(1)}$ & $\eta^{(2)}$ \\
\hline $1 \times 10^{-2}$ & $1 \times 10^{-2}$ & $8.1720 \times 10^{-1}$ & $2.9464 \times 10^{2}$ & $1.1316 \times 10^{5}$ \\
$1 \times 10^{-2}$ & $5 \times 10^{-3}$ & $9.8700 \times 10^{-1}$ & $2.1797 \times 10^{2}$ & $6.6314 \times 10^{4}$ \\
$1 \times 10^{-3}$ & $1 \times 10^{-3}$ & $2.3933 \times 10^{-1}$ & $3.3844 \times 10^{1}$ & $1.2335 \times 10^{4}$ \\
$1 \times 10^{-3}$ & $5 \times 10^{-4}$ & $3.2528 \times 10^{-1}$ & $3.5179 \times 10^{1}$ & $8.1193 \times 10^{3}$ \\
$1 \times 10^{-4}$ & $1 \times 10^{-4}$ & $1.8561 \times 10^{-1}$ & 9.0949 & - \\
$1 \times 10^{-4}$ & $5 \times 10^{-5}$ & $2.6007 \times 10^{-1}$ & $1.7364 \times 10^{1}$ & - \\
$1 \times 10^{-5}$ & $1 \times 10^{-5}$ & $1.8028 \times 10^{-1}$ & $4.5475 \times 10^{2}$ & - \\
\hline
\end{tabular}

Finally, the steady-state mean square error is evaluated using the high-order Kalman filter (22). When $\varepsilon_{1}=\varepsilon_{2}=0.01$, the high-order Kalman filter gain $F_{e}^{\text {exa }}$ is as follows:

$$
F_{e}^{\text {exa }}:=F_{e}^{(20)}=Y_{e}^{(20)} C^{\mathrm{T}} V^{-1}=\Phi_{e}^{-1}\left[\begin{array}{cccc}
7.7511 \times 10^{-1} & -1.2511 \times 10^{-1} & -6.4823 \times 10^{-2} & -5.8349 \times 10^{-2} \\
-1.0570 \times 1 & 6.9146 \times 10^{-1} & 1.7925 \times 10^{-2} & 5.1442 \times 10^{-4} \\
-6.7760 \times 10^{-3} & 1.2926 \times 10^{-2} & -4.6900 \times 10^{-4} & -1.8177 \times 10^{-3} \\
4.1348 \times 10^{-2} & -6.9771 \times 10^{-3} & 2.3353 \times 10^{-2} & -4.9059 \times 10^{-3} \\
7.2956 \times 10^{-1} & -4.1007 \times 10^{-1} & -8.1998 \times 10^{-2} & 2.2135 \times 10^{-2} \\
2.2474 \times 10^{-2} & 1.2294 \times 10^{-2} & 1.9140 \times 10^{-4} & 1.8738 \times 10^{-4} \\
3.2656 \times 10^{-2} & 6.9469 \times 10^{-2} & 4.7563 \times 10^{-5} & 1.0017 \times 10^{-4} \\
-4.5683 \times 10^{-4} & 1.1341 \times 10^{-4} & 2.0714 \times 10^{-2} & 1.4650 \times 10^{-2} \\
-7.9221 \times 10^{-4} & 1.0532 \times 10^{-4} & 2.9300 \times 10^{-2} & 7.2075 \times 10^{-2}
\end{array}\right] .
$$

The near-optimal steady-state mean square per iterations that is, $i=0,1,2$, are given in table 2 , where $\eta^{(i)}=\left(\operatorname{Tr} W_{e}-\operatorname{Tr} Y_{e}\right) /\left(\varepsilon_{1} \varepsilon_{2}\right)^{2 i+1}$. Table 2 verifies that $F_{e}^{(i)}$ has improved the steady-state mean square error as the number of iterations increases. Moreover, it is shown that the property (23) is satisfied because the indices $\eta^{(i)}$ are the same order for the different parameters $\varepsilon_{1}$ and $\varepsilon_{2}$.

When the parameters $\varepsilon_{1}$ and $\varepsilon_{2}$ are less than $0.0001=10^{-4}$, the index $\eta^{(2)}$ is beyond the guaranteed precision because $\left(\varepsilon_{1} \varepsilon_{2}\right)^{-5}$ becomes very large. In that case, it should be noted that in table 2 the index $\eta^{(2)}$ has no value, which is indicated by the symbol - .

\section{Conclusion}

In this paper, the new recursive algorithm for solving the MARE has been proposed. It has been proven that the solution of the MARE converges to a positive semidefinite stabilizing solution with a convergence rate of $O\left(\|\mu\|^{i+1}\right)$. As another important feature, since the assumption that the Hamiltonian matrices $Z_{j j}, j=1,2$, for the fast subsystems have no eigenvalues in common is not needed compared with the work of Coumarbatch and Gajić (2000), the new results are applicable to a more realistic MSPS. Moreover, it is newly proved that the resulting Kalman filter achieves a performance which is $O\left(\|\mu\|^{2 i+1}\right)$, close to the optimal mean square error.

\section{References}

C. Coumarbatch and Z. Gajić, "Parallel optimal Kalman filtering for stochastic systems in multimodeling form", Journal of Dynamic Systems, Measurment, and Centers, 2000, 122, 542-550.

Z. Gajić, "The existence of a unique and bounded solution of the algebraic Riccati equation of multimodel estimation and control problems", Systems and Control Letters, 1988, 10, 185-190.

Z. Gajić, D. Petkovski and X. Shen, Singularly Perturbed and Weakly Coupled Linear System -A Recursive Approach, Lecture Notes in Control and Information Sciences, Berlin: Springer, 1990, Vol. 140.

H.K. Khalil and P.V. Kokotovic, "Control of linear systems with multiparameter singular perturbations", Automatica, 1979, 15, 197-207.

P.V. Kokotović, H.K. Khalil and J. O'Reilly, Singular Perturbation Methods in Control: Analysis and Design, Philadelphia, Pennsylvania: Society for Industrial and Applied Mathematics, 1999.

A.J. Laub, "A Schur method for solving algebraic Riccati equations", IEEE Transactions on Automatic Control, 1979, 24, 913-921. 
J.R. Magnus and H. Neudecker, Matrix Differential Calculus with Applications in Statistics and Econometrics, New York: Wiley, 1999. H. Mukaidani, "Near-optimal Kalman filters for multiparameter singularly perturbed linear systems", IEEE Transactions on Circuits and Systems I, 2003, 50, 717-721.

H. Mukaidani, T. Shimomura and K. Mizukami, "Asymptotic expansions and new numerical algorithm of the algebraic Riccati equation for multiparameter singularly perturbed systems", Journal of Mathematical Analysis and Applications, 2002a, 267, 209-234

H. Mukaidani, T. Shimomura and H. Xu, "Near-optimal control of linear multiparameter singularly perturbed systems", IEEE Transaction on Automatic Control, 2002b, 47, 2051-2057.
H. Mukaidani, T. Shimomura and H. Xu, "Recursive approach of $H_{\infty}$ optimal filtering for multiparameter singularly perturbed systems", Proceedings of the 15th IFAC World Congress, Barcelona, 2002, CD-ROM, 2002c.

H. Mukaidani, H. Xu and K. Mizukami, "Recursive computation of pareto optimal strategy for multiparameter singularly perturbed systems", Dynamic Continuous, Discrete and Impulsive Systems, 2002d, 9b, 175-200.

H. Mukaidani, H. Xu and K. Mizukami, "New results for near-optimal control of linear multiparameter singularly perturbed systems", Automatica, 2003, 39, 2157-2167.

K. Zhou, Essentials of Robust Control, Upper Saddle River, New Jersey: Prentice-Hall, 1998. 